\title{
Degradation and removal of soybean allergen in Japanese soy sauce
}

\author{
NORIHIRO MAGISHI $^{1}$, NAOYA YUIKAWA ${ }^{1}$, MAKIO KOBAYASHI ${ }^{1}$ and SHOICHIRO TANIUCHI $^{2}$ \\ ${ }^{1}$ Research Laboratory, Higashimaru Shoyu Co., Ltd., Tatsuno, Hyogo 679-4167; \\ ${ }^{2}$ Department of Pediatrics, Takatsuki General Hospital, Takatsuki, Osaka 569-1192, Japan
}

Received March 1, 2016; Accepted February 27, 2017

DOI: $10.3892 / \mathrm{mmr} .2017 .6815$

\begin{abstract}
Soy sauce is a traditional fermented seasoning of Japan and is available throughout the world. The two main raw ingredients of soy sauce are soybean and wheat, both of which are established food allergens. The present study examined the degradation and removal of soybean allergens in soy sauce by immunoblotting with anti-soybean protein antibody from rabbit and sera from two children with soybean allergy. It was demonstrated that soybean allergens were gradually degraded during the fermentation process, but were not completely degraded in raw soy sauce. During the processes of heat-treatment and filtration, the soluble soybean allergens in raw soy sauce were denatured to insoluble allergens by heat-treatment and subsequently completely removed from soy sauce by filtration. Therefore, to reduce the allergenicity of soy sauce, heat-treatment and filtration are very important processes in addition to the enzymatic degradation during the fermentation of soy sauce.
\end{abstract}

\section{Introduction}

Soybean is one of the most important sources of dietary protein and lipid worldwide; however, soybean is also one of the commonest food allergens. An immunological study using the sera of patients with soybean allergies identified 16 immunoglobulin (Ig)E-binding components (1). Among them, Gly $\mathrm{m}$ Bd $68 \mathrm{~K}$, Gly $\mathrm{m}$ Bd $30 \mathrm{~K}$ and Gly $\mathrm{m}$ Bd $28 \mathrm{~K}$ proteins in the $7 \mathrm{~S}$ globulin ( $\beta$-conglycinin) fraction are considered to be the major soybean allergens (1). Gly m Bd $68 \mathrm{~K}$ was identified as the $\alpha$-subunit of $\beta$-conglycinin (2-4), which is main storage protein. Gly $\mathrm{m} \mathrm{Bd} 30 \mathrm{~K}$ was demonstrated to be the previously identified $34 \mathrm{kDa}$ soybean seed vacuolar oil-body-associated glycoprotein P34, and a thiol protease family of the papain

Correspondence to: Mr. Norihiro Magishi, Research Laboratory, Higashimaru Shoyu Co., Ltd., 100-3 Tominaga, Tatsuno-cho, Tatsuno, Hyogo 679-4167, Japan

E-mail:nmagishi@higashimaru.co.jp

Key words: allergen, soybean, soy sauce, heat-treatment, filtration, fermentation, degradation, removal superfamily $(1,5)$. Gly $\mathrm{m} \mathrm{Bd} 28 \mathrm{~K}$ is a glycoprotein with an asparagine-linked sugar moiety $(1,6,7)$. In addition, Gly $\mathrm{m} 4$ was reported that is related to cross reaction with pollenosis (8), and oleosin which is oil body membrane protein is reported as major allergen of sesame (9-12) and peanuts (9,12-14).

Soy sauce, or shoyu, is a traditional Japanese fermented seasoning that is available worldwide (15). In Japanese soy sauce, the two primary raw materials, soybeans and wheat, are used in roughly equal amounts. Naturally brewed soy sauce is prepared as presented in Fig. 1 (16-19). Briefly, the soybeans are steamed under conditions of high pressure and temperature, and wheat undergoes roasting and crushing. The soybeans and wheat are combined and a small amount of mold spores (Aspergillus oryzae or A. sojae) is added, and the mixture is subsequently placed on a large, porous plate. To provide optimal conditions for the growth of the mold and enzyme production, temperature- and moisture-controlled air is passed through the plate. Koji is the term used to describe the mold-cultured mixture, and the koji is subsequently mixed with water containing a high concentration of $\mathrm{NaCl}$ to produce a mash, which is termed moromi. The moromi is stored for several months in large tanks at room or an elevated temperature. During storage, the moromi is fermented with lactobacilli and yeasts, and is well aged. Following aging, the moromi is pressed and the liquid portion, which is referred to as raw soy sauce, is pasteurized by heat treatment. The heat-treatment process functions to deactivate microbial enzymes and to insolubilize the residual proteins in the raw soy sauce. The insoluble proteins are completely removed by filtration to obtain the final soy sauce product. During fermentation by microbial proteolytic enzymes, allergens from the raw materials are also degraded into peptides and amino acids; therefore, wheat and major soybean allergens (Gly m Bd 30K and Gly m Bd $28 \mathrm{~K})$ are not detected in soy sauce (19-22). However, soy sauce contains certain bioactive components, in addition to taste and aroma compounds; for example, certain polysaccharides, termed shoyu polysaccharides (SPS), originating from the cell wall of soybeans are resistant to enzymatic hydrolyses and remain even after fermentation (23). These SPS exhibit potent antiallergic activities (23-25), enhancing macrophage and lymphocyte functions (26), iron absorption promotion (27) and hypolipidemic effects (28) in vitro and in vivo.

The present study investigated the degradation of soybean proteins during fermentation and the presence of soybean 
allergens in commercial raw soy sauce in Japan by immunoblot analysis using anti-soybean protein antibody from rabbit and sera from 2 children with soybean allergy. The results demonstrated that soybean allergens remained in the raw soy sauce and were not completely degraded; soybean allergens were detected in 3 out of 7 commercial raw soy sauces in Japan. The soluble soybean allergens in raw soy sauce are denatured to insoluble allergens by heat treatment, and then removed by filtration. Therefore, in addition to the degradation of raw materials during fermentation, heat-treatment and filtration are very important processes for the low allergenicity of soy sauce.

\section{Materials and methods}

Soy sauce. Soy sauce was prepared by Higashimaru Shoyu Co. Ltd. (Tastuno, Japan) as previously described (19). To examine soybean proteins during the brewing of soy sauce, raw soy sauce (salt-soluble fractions of soy sauce) was obtained from each moromi at 2 weeks, 2 months and 6 months after fermentation has begun. The raw soy sauce liquid fraction was pressed from each moromi, and then raw soy sauce was pasteurized by heat-treatment at $>85^{\circ} \mathrm{C}$. The heat-treated soy sauce was centrifuged at $21,500 \mathrm{x} \mathrm{g}$ for 10 min to obtain a supernatant (the heat-treated soy sauce fraction). The solid portion remaining after centrifugation (the heat-denatured sedimentation product) was washed twice with $18 \% \mathrm{NaCl}$, and extracted with $0.1 \mathrm{M}$ Tris- $\mathrm{HCl}$ buffer (pH 8.6) containing $4 \mathrm{M}$ urea for $30 \mathrm{~min}$ (19), followed by centrifugation at $21,500 \mathrm{x}$ g for $10 \mathrm{~min}$ to obtain the heat-denatured sedimentation fraction.

In addition, 7 items of commercial raw soy sauce in Japan (including 4 typical kinds of soy sauce: 'Koikuchi', dark soy sauce; 'usukuchi', light soy sauce; 'tamari', very dark soy sauce; and, 'saishikomi', refermented soy sauce) were also examined. Koikuchi is dark in color and made from equal amounts of soybeans and wheat. Usukuchi is also made from a mixture containing approximately equal amounts of soybeans and wheat; however, rice and gluten may also be added. The principles of the preparation of usukuchi and koikuchi are similar, but all the procedures are directed towards producing a lighter color in the final usukuchi product. Tamari is very dark in color and is made primarily from soybeans with a small quantity of wheat. Saishikomi is made from the moromi mash mixed with both the koji and raw soy sauce instead of more commonly used salt water.

Sera. The present study was approved by the institutional ethics committee of Higashimaru Shoyu Co., Ltd., and informed consent was obtained from each patient or her parents. Sera were obtained from 2 female patients (aged 11 and 9 years) with soybean allergy at the Department of Pediatrics, Kansai Medical University (Hirakata, Japan). The soybean-specific $\mathrm{IgE}$ titers of the patients were 1.01 and $10.9 \mathrm{IU} / \mathrm{ml}$, respectively, as estimated with a radioallergosorbent test (RAST; CAP System FEIA, Pharmacia Diagnostics, Thermo Fisher Scientific, Inc., Uppsala, Sweden), the RAST score was 2-3 for soybean allergy according to the manufacturer's protocol. Sera were used for immunoblotting analysis following 50 fold dilution with distilled water.
Protein electrophoresis and immunoblotting. Proteins were separated by $14 \%$ SDS-PAGEaccording to the methoddescribed by Laemmli (29). To prevent thermal denaturation of samples, $20 \mu \mathrm{l}$ of sample buffer [62.5 mM Tris- $\mathrm{HCl}$ (pH 6.8), 1\% SDS, $5 \%$ 2-mercaptoethanol, $10 \%$ glycerol and $0.01 \%$ bromophenol blue] was added to $80 \mu \mathrm{l}$ of soy sauce samples and incubated at $37^{\circ} \mathrm{C}$ overnight. The denatured sample was applied in $10 \mu \mathrm{l}$ aliquots and the molecular weight marker used was Protein Molecular Weight Marker (Low; 3450; Takara Bio, Inc., Shiga, Japan). The gel was divided into 2 parts. Proteins separated on one part of the gel were stained with Coomassie Brilliant Blue R-250 dye (Quick-CBB for electrophoresis; Wako Pure Chemical Industries, Ltd., Osaka, Japan) and those separated on the other part of the gel were transferred electrophoretically onto a polyvinylidene difluoride membrane (AE-6666; Atto Corporation, Tokyo, Japan) for $1 \mathrm{~h}$ at a fixed current of $100 \mathrm{~mA}$. For immunoblotting of soybean protein, the membrane was blocked overnight with $1 \%$ bovine serum albumin (BSA; FIA/RIA grade, essentially globulin free; Nacalai Tesque, Inc., Kyoto, Japan) in PBS-Tween-20 (0.1\%; Sigma-Aldrich; Merck KGaA, Darmstadt, Germany) at $4^{\circ} \mathrm{C}$. The proteins on the membrane were probed with primary rabbit anti-soybean protein polyclonal antibody (S2519; 1:5,000; Sigma-Aldrich; Merck KGaA) diluted with PBS-Tween-20 at $25^{\circ} \mathrm{C}$ overnight. After washing for 30 min with $1 \%$ BSA in PBS-Tween-20 $(0.1 \%)$, the membrane was incubated with secondary biotinylated goat anti-rabbit IgG (PK-4001; Vectastain ${ }^{\circledR}$ ABC HRP Rabbit IgG kit; Vector Laboratories, Inc., Burlingame, CA, USA) following 5,000 fold dilution with PBS-Tween-20 (0.1\%) at $25^{\circ} \mathrm{C}$ for $1 \mathrm{~h}$, according to the manufacturer's protocol. After washing for 30 min with $1 \%$ BSA in PBS-Tween-20 (0.1\%), the membrane was incubated with an avidin-biotinylated enzyme complex-peroxidase (PK-4001; Vectastain ${ }^{\circledR}$ ABC HRP Rabbit IgG kit; Vector Laboratories, Inc.) at $25^{\circ} \mathrm{C}$ for $30 \mathrm{~min}$. After washing for $30 \mathrm{~min}$ with $1 \% \mathrm{BSA}$ in PBS-Tween-20 (0.1\%), detection was performed by the addition of 3,3'-diaminobenzidine tetrahydrochloride (DAB; SK-4100; DAB Peroxidase HRP Substrate kit; Vector Laboratories, Inc.), according to the manufacturer's protocol.

For immunoblotting of soybean allergen, the membrane (AE-6666; Atto Corporation) was blocked for $3 \mathrm{~h}$ with $1 \% \mathrm{BSA}$ in PBS-Tween-20 $(0.1 \%)$ at $4^{\circ} \mathrm{C}$. The proteins on the membrane were probed with the sera of patients at $25^{\circ} \mathrm{C}$ for $3 \mathrm{~h}$. After washing for 30 min with $1 \%$ BSA in PBS-Tween-20 (0.1\%), the membrane was incubated with biotinylated goat anti-human IgE (BA-3040; Vector Laboratories, Inc.) at $25^{\circ} \mathrm{C}$ for $1 \mathrm{~h}$, according to the protocol of this kit. After washing for $30 \mathrm{~min}$ with PBS-Tween-20 (0.1\%), the membrane was incubated with the avidin-biotinylated enzyme complex-peroxidase at $25^{\circ} \mathrm{C}$ for $30 \mathrm{~min}$, and subsequently washed for $30 \mathrm{~min}$ with $1 \% \mathrm{BSA}$ in PBS-Tween-20 (0.1\%). Detection was performed by the addition of DAB. All experiments were repeated $\geq 2$ times.

\section{Results and Discussion}

Soybean protein degradation during soy sauce fermentation. To examine the degradation of soybean proteins during soy sauce fermentation, immunoblotting analyses of raw soy sauce from each moromi at 2 weeks, 2 months and 6 months of fermentation were performed using rabbit anti-soybean 


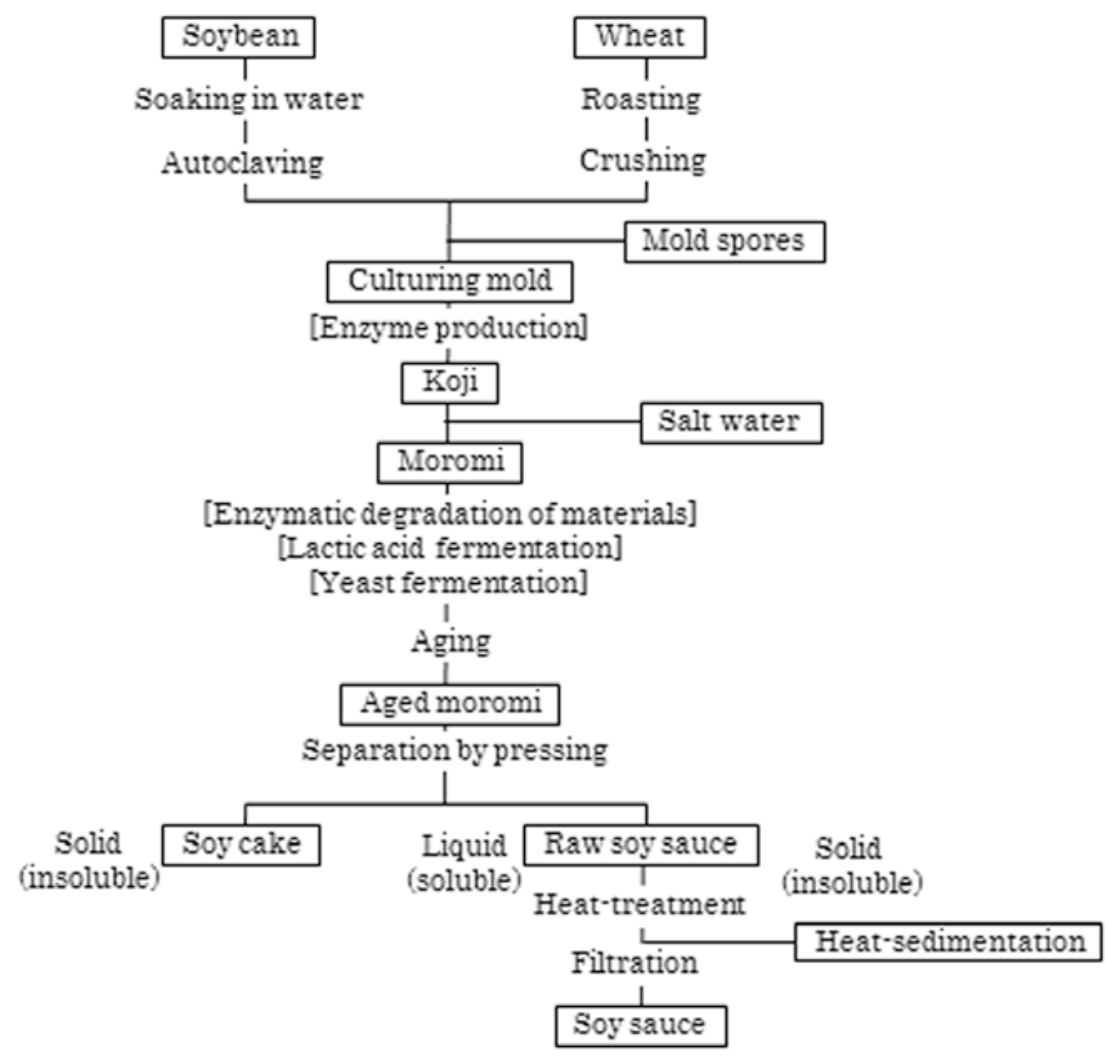

Figure 1. Brewing process of soy sauce from raw materials to the final product.

A

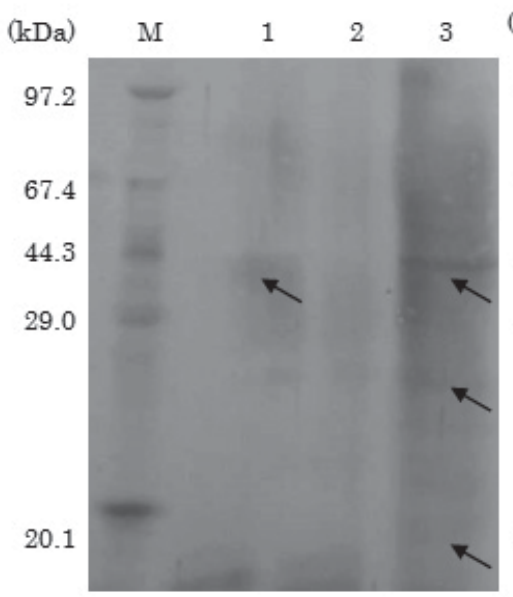

B

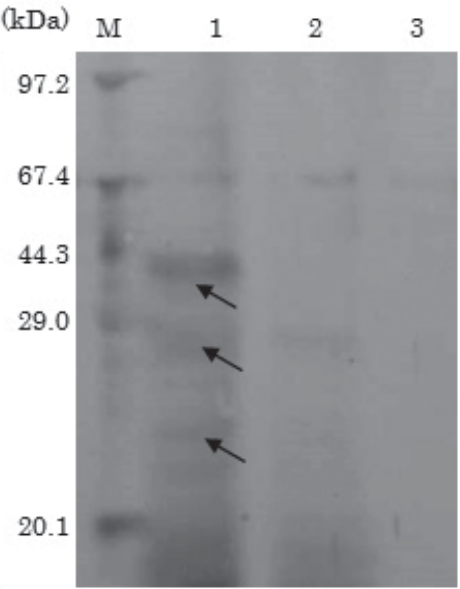

C

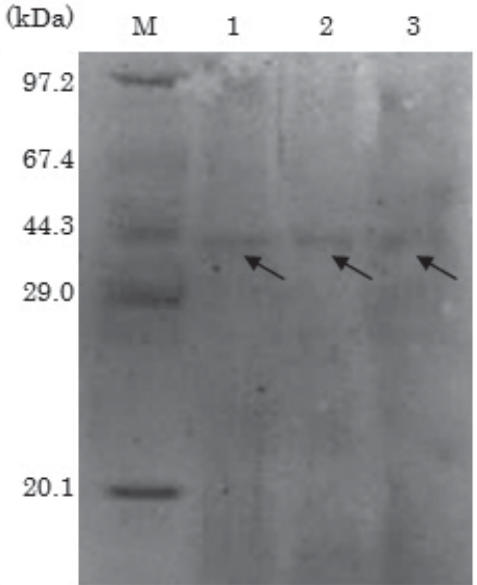

Figure 2. Immunoblotting analysis was performed on moromi at different durations of fermentation and stages of processing. Immunoblotting analysis of (A) raw soy sauce, (B) heat-treated soy sauce and (C) the sedimentation fraction. Samples were added to the sample buffer and incubated at $37^{\circ} \mathrm{C}$ overnight. Aliquots were subjected to electrophoresis and immunoblot analysis. Lane M, marker proteins; lane 1, 2 weeks fermentation; lane 2, 2 months fermentation; lane 3, 6 months fermentation of moromi. Arrows indicate detected soybean protein (e.g., Gly m Bd 30K and partially degraded proteins). Background levels are considered to be due to the presence of partially degraded proteins during the fermentation process in the different samples.

protein antibody. Soybean proteins from the raw materials are gradually degraded and solubilized during fermentation, but Gly $\mathrm{m} \mathrm{Bd} 30 \mathrm{~K}$ and partially degraded soybean proteins remained in raw soy sauce of the 6 months moromi and soybean proteins were not completely degraded even after fermentation (Fig. 2A). Following heat treatment $\left(85^{\circ} \mathrm{C}\right)$ and filtration to separate it into the heat-treated soy sauce and the heat-denatured sedimentation fraction, no soybean protein was detected in the heat-treated soy sauce of the 6 months moromi, although soybean proteins (e.g., Gly m Bd $30 \mathrm{~K}$ and partly degraded proteins) were detected in the heat-treated soy sauce of the 2 weeks and 2 months moromi (Fig. 2B). It was regarded as an effect of thermal denaturation that more soybean protein was detected in raw soy sauce compared with 


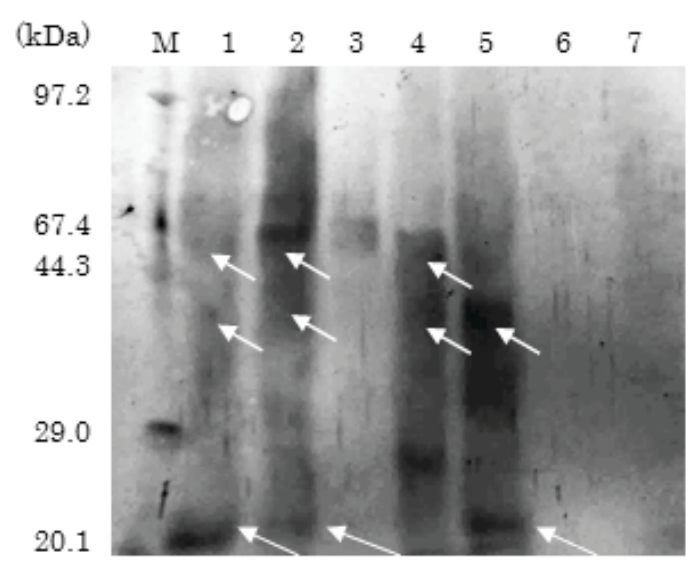

Figure 3. Immunoblotting analysis of commercial raw soy sauce using rabbit anti-soybean protein antibody. Samples were added to the sample buffer and incubated at $37^{\circ} \mathrm{C}$ overnight. Aliquots were subjected to electrophoresis and immunoblot analysis. Lane: M, marker proteins; lanes 1-4, koikuchi; lane 5, saishikomi; lane 6, tamari; lane 7, usukuchi. Arrows indicate detected soybean protein (e.g., Gly m Bd 30K and partially degraded proteins).

A

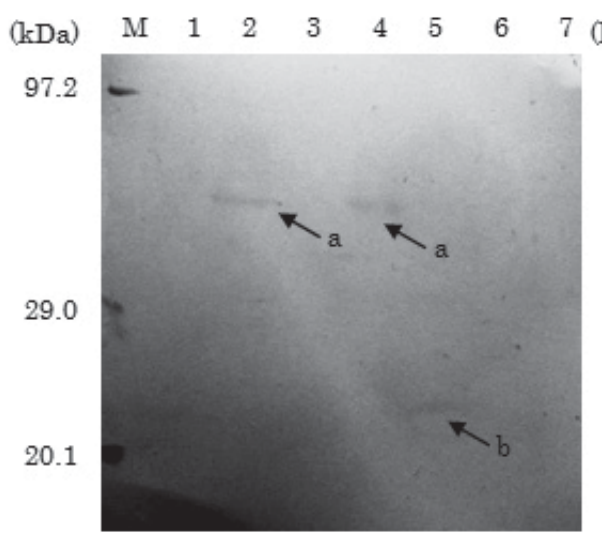

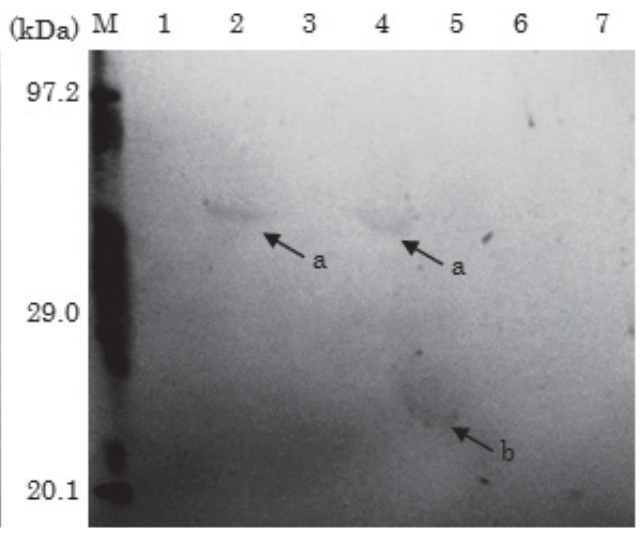

Figure 4. Immunoblotting analysis of commercial raw soy sauce using the sera from patients with soybean allergy. Immunoblotting analysis using sera from either (A) an 11 year-old girl with a soybean-specific IgE titer of $1.01 \mathrm{UA} / \mathrm{ml}$ and a RAST score of 2, or (B) a 9-year old girl with a soybean-specific IgE titer of $10.9 \mathrm{UA} / \mathrm{ml}$ and a RAST score of 3. Arrows labeled 'a' and ' $\mathrm{b}$ ' indicate the predicted $\beta$-subunit of $\beta$-conglycinin and oleosin soybean allergens detected in raw soy sauce, respectively.

heat-treated soy sauce. In the heat-sedimentation fraction, soybean proteins were detected in the moromi at 2 weeks, 2 months and 6 months (Fig. 2C), indicating that the soluble soybean proteins were denatured to insoluble soybean proteins by heat-treatment and then removed completely by filtration.

Soybean allergens in commercial raw soy sauce. The majority of soy sauce produced in Japan is heat-treated; however, a certain amount of raw soy sauce is also produced. The present study investigated 7 commercially available raw soy sauce in Japan. Immunoblot analysis with the rabbit anti-soybean protein antibody detected soybean proteins in 4 of the 7 sauces, including 3 types of koikuchi (lanes 1,2 and 4) and the saishikomi (lane 5; Fig. 3). Immunoblotting with the sera from two children with soybean allergy, detected soybean allergens in 3 of the 7 soy sauces, including 2 of the koikuchi (lanes 2 and 4) and the saishikomi (lane 5; Fig. 4). The molecular weight of soybean allergen detected in koikuchi of lane 2 and 4 was $\sim 50 \mathrm{kDa}$ and it was predicted to be the $\beta$-subunit of $\beta$-conglycinin. The soybean allergen detected in saishikomi of lane 5 was $\sim 23 \mathrm{kDa}$ and predicted to be oleosin. Gly $\mathrm{m} \mathrm{Bd}$
$30 \mathrm{~K}$ and Gly m Bd 28K were not detected, consistent with the results of previous studies (20-22).

In the current study, by using immunoblotting with the anti-soybean antibody and the sera from 2 children with soybean allergy, it was clearly demonstrated that soybean protein was not completely degraded in moromi and remained in the raw soy sauce. Furthermore, the soluble soybean proteins that remained in the raw soy sauce were denatured to insoluble allergens by heat-treatment and were completely removed from 6 month moromi by subsequent filtration. Soybean proteins were detected in certain commercial raw soy sauces (Fig. 3), and these soybean proteins were identified to be soybean allergens as they were immunostained by the sera from 2 children with soybean allergy. Notably, soybean allergens detected in commercial raw soy sauce were predicted to be the $\beta$-subunit of $\beta$-conglycinin and oleosin, which are not known as major soybean allergens. The $\beta$-subunit of $\beta$-conglycinin is highly homologous with the $\alpha$-subunit, which is a major soybean allergen (3). Oleosin is a hydrophobic protein that is located in the outer layer of the oil body of plant cells, and is reported as an allergen in sesame (9-12) and peanuts (9,12-14). In conclusion, for soy 
sauce to have low allergenicity, heat-treatment and filtration are important processes for the removal soybean allergens, in addition to the enzymatic degradation in the moromi fermentation.

\section{References}

1. Ogawa T, Bando N, Tsuji H, Sasaoka K, Nishikawa K and Sasaoka K: Investigation of the IgE-binding proteins in soybeans by immunoblotting with the sera of the soybean-sensitive patients with atopic dermatitis. J Nutr Sci Vitaminol (Tokyo) 37: 555-565, 1991.

2. Ogawa T, Bando N, Tsuji H, Nishikawa K and Kitamura K: Alpha-subunit of beta-conglycinin, an allergenic protein recognized by $\operatorname{IgE}$ antibodies of soybean-sensitive patients with atopic dermatitis. Biosci Biotechnol Biochem 59: 831-833, 1995.

3. Krishnan HB, Kim WS, Jang S and Kerley MS: All three subunits of soybean beta-conglycinin are potential food allergens. J Agri Food Chem 57: 938-943, 2009.

4. Adachi A, Horikawa T, Shimizu H, Sarayama Y, Ogawa T, Sjolander S, Tanaka A and Moriyama T: Soybean beta-conglycinin as the main allergen in a patient with food-dependent exercise-induced anaphylaxis by tofu: Food processing alter pepsin resistance. Clin Exp Allergy 39: 167-173, 2009.

5. Ogawa T, Tsuji H, Bando N, Kitamura K, Zhu YI, Hirano H and Nishikawa K: Identification of the soybean allergenic protein, Gly $\mathrm{m} \mathrm{Bd} 30 \mathrm{~K}$, with the soybean seed 34-kDa oil-body-associated protein. Biosci Biotechnol Biochem 57: 1030-1033, 1993.

6. Tsuji H, Bando N, Hiemori M, Yamanishi R, Kimoto M, Nishikawa $\mathrm{K}$ and Ogawa T: Purification of characterization of soybean allergen Gly m Bd 28K. Biosci Biotechnol Biochem 61: 942-947, 1997.

7. Tsuji H, Hiemori M, Kimoto M, Yamashita H, Kobatake R, Adachi M, Fukuda T, Bando N, Okita M and Utsumi S: Cloning of cDNA encoding a soybean allergen, Gly $\mathrm{m} \mathrm{Bd} 28 \mathrm{~K}$. Biochim Biophys Acta 1518: 178-182, 2001.

8. Kleine-Tebbe J, Vogel L, Crowell DN, Haustein UF and Vieths S: Severe oral allergy syndrome and anaphylactic reactions caused by a Bet v 1- related PR-10 protein in soybean, SAM2. J Allergy Clin Immunol 110: 797-804, 2002.

9. Morisset M, Moneret-Vautrin DA, Kanny G, Guénard L, Beaudouin E, Flabbée J and Hatahet R: Thresholds of clinical reactivity to milk, egg, peanut and sesame in immunoglobulin E-dependent allergies: Evaluation by double-blind or single-blind placebo-controlled oral challenges. Clin Exp Allergy 33: 1046-1051, 2003.

10. Chen JC, Lin RH, Huang HC and Tzen JT: Cloning, expression and isoform classification of a minor oleosin in sesame oil bodies. J Biochem 122: 819-824, 1997.

11. Leduc V, Moneret-Vautrin DA, Tzen JT, Morisset M, Guerin L and Kanny G: Identification of oleosins as major allergens in sesame seed allergic patients. Allergy 61: 349-356, 2006.

12. Pons L, Olszewski A and Guéant JL: Characterization of the oligomeric behavior of a $16.5 \mathrm{kDa}$ peanut oleosin by chromatography and electrophoresis of the iodinated form. J Chromatogr B Biomed Sci Appl 706: 131-140, 1998.

13. Huang AHC: Oil bodies and oleosins in seeds. Ann Rev Plant Physiol Plant Mol Biol 43: 177-200, 1992.

14. Pons L, Chery C, Romano A, Namour F, Artesani MC and Guéant JL: The $18 \mathrm{kDa}$ peanut oleosin is a candidate allergen for IgE-mediated reactions to peanuts. Allergy 57 (Suppl 72): S88-S93, 2002.
15. Yokotsuka T: Soy sauce biochemistry. Adv Food Res 30: 195-329, 1986.

16. Kobayashi M and Hayashi S: Modeling combined effects of temperature and $\mathrm{pH}$ on growth of Zygosaccharomyces rouxii in soy sauce mash. J Ferment Bioeng 85: 638-641, 1998.

17. Kobayashi M and Hayashi S: Supplementation of $\mathrm{NaCl}$ to starter culture of the soy yeast Zygosaccharomyces rouxii. J Ferment Bioeng 85: 642-644, 1998.

18. Nagai H, Kobayashi M, Tsuji Y, Nakashimada Y, Kakizono T and Nishio N: Biological and chemical treatment of solid waste from soy sauce manufacture. Water Sci Technol 45: 335-338, 2002.

19. Kobayashi M, Hashimoto $Y$, Taniuchi S and Tanabe S: Degradation of wheat allergen in Japanese soy sauce. Int J Mol Med 13: 821-827, 2004.

20. Tsuji H, Okada N, Yamanishi R, Bando N, Kimoto M and Ogawa T: Measurement of Gly m Bd 30K, a major soybean allergen, in soybean products by a sandwich enzyme-linked immunosorbent assay. Biosci Biotechnol Biochem 59: 150-151, 1995.

21. Ogawa T, Samoto M and Takahashi K: Soybean allergens and hypoallergenic soybean products. J Nutr Sci Vitaminol (Tokyo) 46: 271-279, 2000.

22. Bando N, Tsuji H, Hiemori M, Yoshimizu K, Yamanishi R, Kimoto M and Ogawa T: Quantitative analysis of Gly $\mathrm{m} \mathrm{Bd} 28 \mathrm{~K}$ in soybean products by a sandwich enzyme-linked immunosorbent assay. J Nutr Sci Vitaminol (Tokyo) 44: 655-664, 1998.

23. Kobayashi M, Matsushita H, Yoshida K, Tsukiyama R, Sugimura $\mathrm{T}$ and Yamamoto K: In vitro and in vivo anti-allergic activity of soy sauce. Int J Mol Med 14: 879-884, 2004.

24. Kobayashi M, Matsushita H, Shioya I, Nagai M, Tsukiyama R, Saito M, Sugita T, Sugimura T and Yamamoto K: Quality of life improvement with soy sauce ingredients, shoyu polysaccharides, in perennial allergic rhinitis: A double-blind placebo-controlled clinical study. Int J Mol Med 14: 885-889, 2004.

25. Kobayashi M, Matsushita H, Tsukiyama R, Saito M and Sugita T: Shoyu polysaccharides from soy sauce improve quality of life for patients with seasonal allergic rhinitis: A double-blind placebo-controlled clinical study. Int J Mol Med 15: 463-467, 2005.

26. Matsushita H, Kobayashi M, Tsukiyama R and Yamamoto K: In vitro and in vivo immunomodulating activities of Shoyu polysaccharides from soy sauce. Int J Mol Med 17: 905-909, 2006.

27. Kobayashi M, Nagatani Y, Magishi N, Tokuriki N, Nakata Y, Tsukiyama R, Imai H, Suzuki M, Saito M and Tsuji K: Promotive effect of Shoyu polysaccharides from soy sauce on iron absorption in animals and humans. Int J Mol Med 18: 1159-1163, 2006

28. Kobayashi M, Magishi N, Matsushita H, Hashimoto T, Fujimoto M, Suzuki M, Tsuji K, Saito M, Inoue E, Yoshikawa Y and Matsuura T: Hypolimidemic effect of Shoyu polysaccharides from soy sauce in animals and humans. Int J Mol Med 22: 565-570, 2008 .

29. Laemmli UK: Cleavage of structural proteins during the assembly of the head of bacteriophage T4. Nature 227: 680-685, 1970. 